\title{
Schwarzschild Models for the Galaxy
}

\author{
Julio Chanamé \\ Carnegie Institution of Washington, Department of Terrestrial Magnetism \\ 5241 Broad Branch Rd., Washington DC 20015, USA \\ email: jchaname@dtm.ciw.edu
}

\begin{abstract}
Schwarzschild's orbit-superposition technique is the most developed and well-tested method available for constraining the detailed mass distributions of equilibrium stellar systems. Here I provide a very short overview of the method and its existing implementations, and briefly discuss their viability as a tool for modeling the Galaxy using Gaia data.
\end{abstract}

\section{Introduction}

Models are used to relate observations to theoretical constructs such as the phasespace distribution function. A number of simplifying assumptions are required to make the modeling process tractable and these assumptions can have profound implications for the inferred mass distribution. A delicate balance must be struck between the available observations and the complexity of the models fitted to them.

The Gaia mission will require modeling techniques that are capable of handling huge numbers of measurements, while taking full advantage of the high precision of the data. Existing implementations of the orbit-superposition method already fulfil some of these requirements but do have limitations. For example, while an assumption regarding the geometry of the gravitational potential is inescapable, Schwarzschild models can be built that are completely free from assumptions regarding the detailed orbital structure, which generally have the largest impact on the derived mass distribution.

\subsection{Schwarzschild's technique}

Given an orbit library for an assumed gravitational potential, the orbit-superposition technique (Schwarzschild 1979) finds the linear sum of those orbits that best reproduces the available observations. The success of the method relies on two aspects: (1) that the stellar system can be safely considered to be in equilibrium, and (2) that the orbit library is sufficiently comprehensive. If these two conditions are satisfied, the method is very general and free from most assumptions. Even the required assumption of a given geometry for the gravitational potential is in practice removed by the iterative nature of the technique, which calls for the construction of Schwarzschild models for an entire grid of potentials, with the final model the one that best fits the data. Of course a Schwarzschild model only provides a snapshot of the current dynamical state of the system, and the question of stability must be addressed by other means.

Schwarzschild models have been successfully used to constrain the dark-matter halos of galaxies (e.g., Rix et al. 1997, Thomas et al. 2005), to weigh supermassive black holes at the centers of both galaxies (e.g., van der Marel et al. 1998, Gebhardt et al. 2000, 2001) and globular clusters (Gebhardt, Rich, \& Ho 2005). They have also been used to study the dynamics of star clusters (e.g., van de Ven et al. 2006). More relevant to the subject at hand, orbit-superposition models have also been used to study the dynamics of the Galactic bulge (Zhao 1996, Häfner et al. 2000). Existing implementations of the Schwarzschild method are usually classified/labeled according to the geometry of the 
stellar systems they can be applied to (spherical, axisymmetric, triaxial), and to the type of dataset they are designed to handle (continuous or discrete; see Chanamé, Kleyna, \& van der Marel 2008 for a review).

Points of weakness or controversy regarding Schwarzschild modeling include: the nonuniqueness of the initial conditions used to generate orbits; the amount of smoothing or regularization of the solution that is applied and its impact on the final results; possible over-interpretation of $\chi^{2}$ plots and indeterminacy of best solution; how to deal with incomplete positional sampling; and large computational costs.

\section{Applicability of Schwarzschild's method to Galactic surveys}

We can consider the Galaxy to be composed of two kinds of structure: (1) a smoothly distributed and old Galaxy in steady-state equilibrium, and (2) a perturbed, inhomogeneous Galaxy that changes over relatively short timescales and is not in dynamical equilibrium. While the classical Galactic structures of bulge, disk(s), and halo belong to the first category, shorter-lived structures such as tidal streams, spiral arms, and disk warps, all fall into the second one. Only the background, steady-state Galaxy is susceptible to Schwarzschild modeling. Fortunately, most of the Galaxy's mass lies in steady-state structures, so a Schwarzschild model should provide a useful first approximation to the data. However, even though the non-equilibrium mass fraction is small, this component is expected to hold clues to the history of the Galaxy, and means must be found to model it too.

Modeling data from current surveys such as SDSS and RAVE will prepare us for modeling the vastly superior Gaia data. Clever arguments such as those in Smith, Evans, \& An (2009) can only benefit the applicability of Schwarzschild's technique by narrowing down the range of possible shapes and geometries of the underlying gravitational potential, and could even shed light on the optimal choice of initial conditions for orbit integration.

\section{Acknowledgements}

I thank the American Astronomical Society and the International Astronomical Union for travel grants. I acknowledge support from NASA through Hubble Fellowship grant HF-01216.01-A, awarded by the Space Telescope Science Institute, which is operated by the AURA, Inc., under NASA contract NAS5-26555.

\section{References}

Chanamé, J., Kleyna, J., \& van der Marel, R. 2008, ApJ 682,841

Gebhardt, K., Richstone, D., Kormendy, J., et al. 2000, AJ 119,1157

Gebhardt, K., Lauer, T., Kormendy, J., et al. 2001, AJ 122,2469

Gebhardt, K., Rich, M., \& Ho, L. 2005, ApJ 634,1093

Häfner, R., Evans, N. W., Dehnen, W., \& Binney, J. 2000, MNRAS 314,433

Rix, H.-W., et al., 1997, ApJ 488,702

Schwarzschild, M. 1979, ApJ 232,236

Smith, M. C., Wyn Evans, N., \& An, J. H. 2009, ApJ 698,1110

Thomas, J., et al., 2005, MNRAS 360,1355

van de Ven, G., van den Bosch, R. C. E., Verolme, E. K., \& de Zeeuw, P. T. 2006, A\& A 445,513

van der Marel, R. P., Cretton, N., de Zeeuw, P. T., \& Rix, H.-W. 1998, ApJ 493,613

Zhao, H. S. 1996, MNRAS 283,149 